\title{
Multiperiodic Variability and Outbursts of 28 Cygni
}

\author{
S. Tubbesing, Th. Rivinius, B. Wolf
}

Landessternwarte Königstuhl, D-69117, Heidelberg, Germany

\author{
A. Kaufer \\ European Southern Observatory, Santiago, Chile
}

\begin{abstract}
Cygni was observed during two seasons spectroscopically. The radial velocities of more than 15 spectral lines were measured and searched for periodic variability. Two closely spaced periods were found. A two-dimensional analysis of the line profile variability yielded the same periods and provided the power and phase distribution across the line profiles. An outburst covered by our data coincides with the time of constructive interference of both periods. $28 \mathrm{Cyg}$ is the second case for which outbursts seem to be triggered by multi-mode beating.
\end{abstract}

\section{Introduction}

Recent reports on multiperiodicity of $\mu$ Cen have shown strong evidence for a connection between this property and the timing of circumstellar line-emission outbursts (e.g. Rivinius et al. 1998b). A project has been started to monitor northern Be stars over the span of several years to find further cases of such a behaviour. $28 \mathrm{Cyg}$ was included due to earlier reports on multiperiodicity (e.g. Bossi et al., 1993). The monitoring was done with HERos (Štefl \& Rivinius, this volume) for 100 days (104 spectra) at Heidelberg in 1997 and 65 days (105 spectra) with the $1.23 \mathrm{~m}$ telescope at Calar Alto in 1998, respectively. In 1997 only minor emission variability was detected, but no long-term trend. Yet the star showed a significantly different appearance in 1998. Therefore it must have undergone major variations between the runs. Fig. 1 shows the mean profiles from both seasons for several spectral lines.

\section{Periodic variability}

The radial velocities (RV's) of the line centers (modes) of more than $15 \mathrm{He}$ I lines were obtained by fitting a Gaussian interactively to the very core of the line. On the averaged RV data a period analysis was performed using Scargle's method (Table 1). The resulting power spectra are displayed in Fig. 2. To ascertain the reality of the found peaks a further $2 \mathrm{D}$ analysis was done. The two clearly strongest peaks at $\mathcal{P}_{1}=0.64699$ day and $\mathcal{P}_{2}=0.62487$ day passed this test, any others did not yield a meaningful variability pattern across the line profile (Fig. 3). To get a stronger signal from the weak variability connected to $\mathcal{P}_{2}$ we 

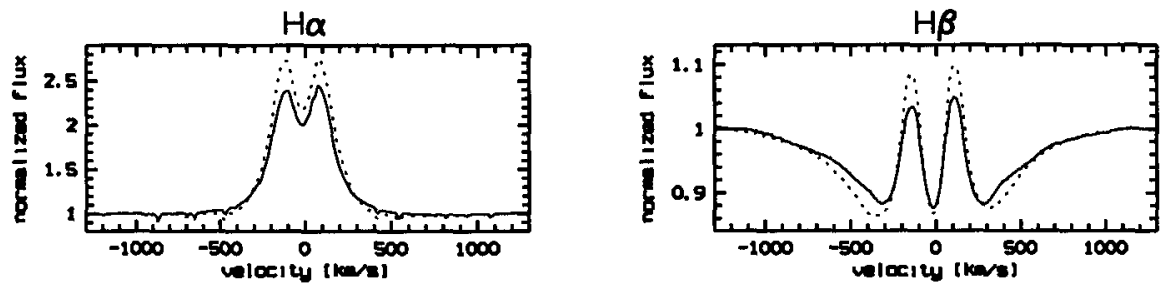

HeI $\lambda 6678$

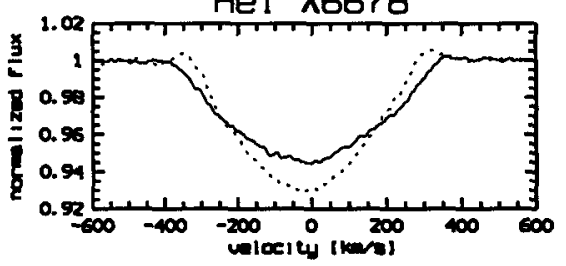

Fel I $\lambda 4583$

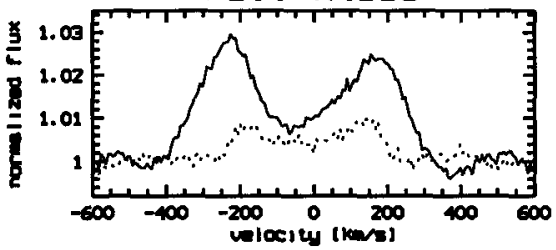

Figure 1. Mean profiles from both seasons (dotted 1997, solid 1998) of the $\mathrm{H} \alpha, \mathrm{H} \beta, \mathrm{He}$ I $\lambda 6678$ and $\mathrm{Fe}$ II $\lambda 4583$ line of $28 \mathrm{Cyg}$. The emission intensity decreases for $\mathrm{H} \alpha$ and $\mathrm{H} \beta$, while it increases for Fe II $\lambda 4583$. The wings of the Balmer lines increase, however. A gain in peak separation and base width is apparent also for Fe II $\lambda 4583$

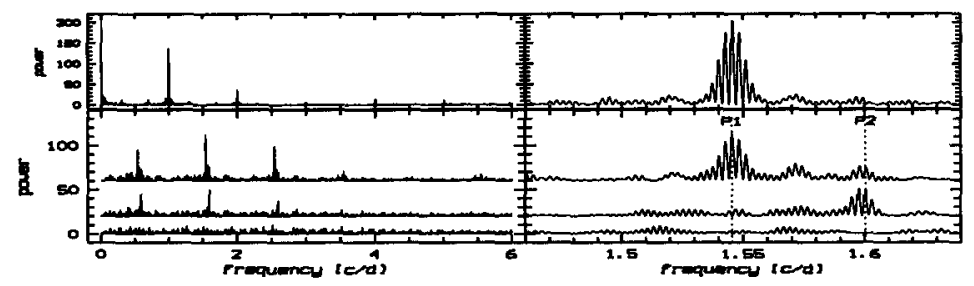

Figure 2. Window function (upper panel) and power spectra of the RV's of all HeI lines measured. The region around the found periods is shown at the right with the windowfunction centered on $\mathcal{P}_{1}$. The original power spectrum and after prewhitening for the periods $\mathcal{P}_{1}$ and $\mathcal{P}_{2}$ are shown in the lower panel from top to bottom

combined 5 HeI lines into a mean profile for each spectrum and performed the analysis on this dataset. The variability pattern of both periods are shown in Fig. 3 for several absorption lines. The observed pattern exhibit a double-peaked power distribution and a smooth phase propagation with a phase difference from blue to red of the order of 1 to $2 \pi$. Photospherically formed lines vary in phase for each period. By these properties the observed variability is similar to patterns of other Be stars like $\mu$ Cen (Rivinius et al. 1998b) and $\omega$ (28) CMa (Maintz et al. this volume). 
Table 1. Results of the period analysis of HeI RV's

\begin{tabular}{c|ccc} 
& $\begin{array}{c}\text { Period } \\
\text { [day] }\end{array}$ & $\begin{array}{c}\text { Amplitude } \\
{[\mathrm{km} / \mathrm{s}]}\end{array}$ & $\begin{array}{c}\text { Phase at JD } \\
2450000.5\end{array}$ \\
\hline $\mathcal{P}_{1}$ & $0.64699 \pm 0.00003$ & $20.6 \pm 1.6$ & $0.40 \pm 0.02$ \\
$\mathcal{P}_{2}$ & $0.62487 \pm 0.00005$ & $10.1 \pm 1.5$ & $0.79 \pm 0.03$
\end{tabular}

\section{Outburst type variability}

Unfortunately we only can point out the differences from 1997 to 1998 (Fig. 1) because of the seasonal gap. However, the observed features fit well the picture of a strong line emission outburst as described by Rivinius et al. (1998a) for $\mu$ Cen. Furthermore we detected signs of a minor outburst during our 1998 observations (Fig. 4). The date of the high combined (beat) amplitude of $\mathcal{P}_{1}$ and $\mathcal{P}_{2}$ coincides with this event. However, other times of high amplitude occurences, every 18.2 days, are not accompanied by an outburst, although irregularities in the equivalent width of $\mathrm{H} \alpha$ might indicate enhanced activity (cf. Fig. 4).
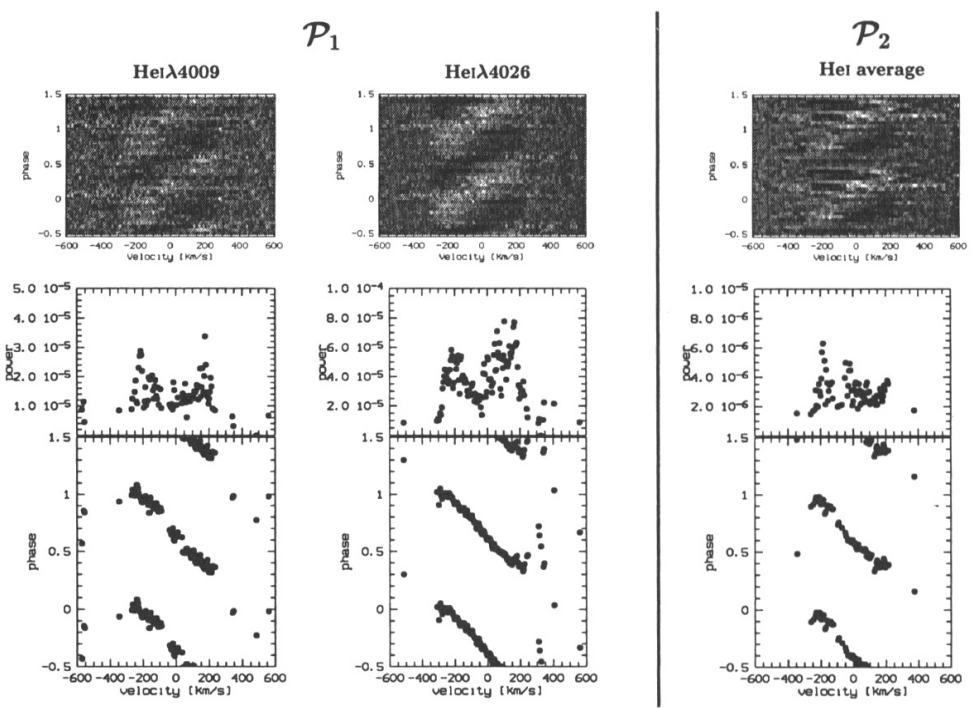

Figure 3. Spectral variability pattern of $28 \mathrm{Cyg}$. The pictures in the upper panels are dynamical spectra of two full pulsation cycles in different spectral lines presented as residuals from the respective mean profile. The lower row shows the results of the two-dimensional time series analysis of the line fluxes in bins of $5 \mathrm{~km} \mathrm{~s}^{-1}$ width. Due to the lower power of $\mathcal{P}_{2}$ a mean profile out of $5 \mathrm{HeI}$ lines $(\lambda \lambda 4009,4026$, $4143,4387,4713$; rightmost panel) was derived to increase the $S / N$ ratio for the $2 \mathrm{D}$ analysis 


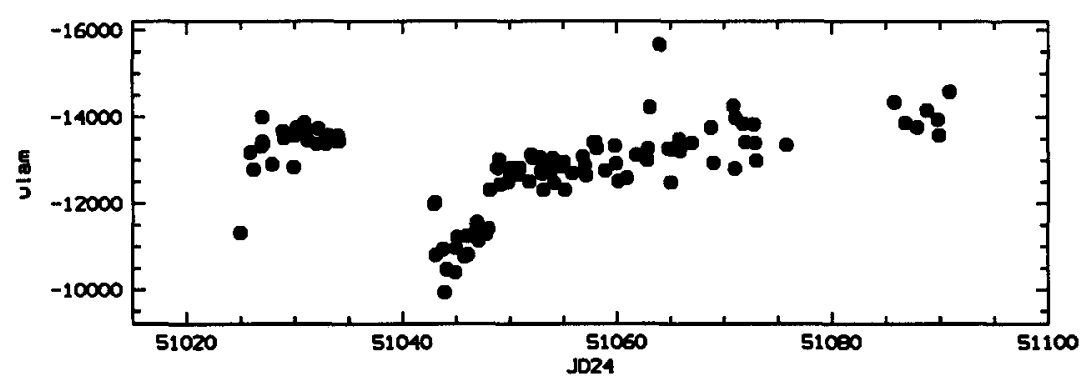

Figure 4. The minor outburst in 1998 can be well detected by the sudden weakening and re-strengthening of the $\mathrm{H} \alpha$ emission (Rivinius et al. 1998a). Judging from equivalent width, enhanced activity could also be present at $\mathrm{MJD}=51025$ and 51063 , about 19 days before and after the clear minor outburst at 51043

\section{Conclusions}

Biperiodicity of the main period of 0.64 day was found, but no further periods. The properties of both periods are nearly identical, i.e. the periods differ by only $3.5 \%$ and have the same appearance, except a difference in total variation power. In shorter datasets the beating might appear as a single period of variable amplitude. 28 Cyg shows enhanced circumstellar activity up to line emission outbursts at times of high co-added amplitude. This confirms the applicability of the outburst timing mechanism found in $\mu$ Cen to further Be stars. Multiperiodic beating leading to outbursts is not an exotic special case, but at least one of the regular mechanisms closely connected to the Be phenomenon. Concerning both power- and phase-distribution across the profile, as well as by small frequency spacing of only $3.5 \%$, the observed properties of the periodicities of 28 Cyg are very similar to the lower period group in $\mu$ Cen. Especially the coincidence of a minor outburst event with the amplitude maximum in 28 Cyg is an important piece of evidence that the timing mechanism for outbursts in $\mu$ Cen is not an exotic one. The same line profile variability mechanism as in $\mu$ Cen and $\omega$ (28) $\mathrm{CMa}$, non-radial pulsation, might act in $28 \mathrm{Cyg}$ as well (Rivinius, this volume, Maintz et al. this volume).

Acknowledgments. ST thanks the Astronomische Gesellschaft for financial support to attend the meeting.

\section{References}

Bossi, M., Guerrero, G., Zanin, F. 1993, A\&A 269, 343

Rivinius, Th., Baade, D., Štefl S, et al. 1998a, A\&A 333, 125

Rivinius, Th., Baade, D., Štefl S, et al. 1998b, A\&A 336, 177 